\title{
Pedestrian Systems Modelling: Opportunities and Challenges
}

\author{
Martha A. Centeno, $\mathrm{PhD}^{1}$, Lindsay Álvarez Pomar, $\mathrm{MSc}^{2}$, and German Méndez Giraldo, $\mathrm{PhD}^{1}$ \\ ${ }^{1}$ Universidad del Turabo, Puerto Rico; Universidad Distrital Francisco José de Caldas, Colombia; \\ centenom1@suagm.edu, gmendez@udistrital.edu.co. \\ ${ }^{2}$ Universidad Distrital Francisco José de Caldas, Colombia, lalvarez@udistrital.edu.co
}

\begin{abstract}
Designing pedestrian systems have been a challenge due to the lack of effective tools and methods to model the autonomous behavior of pedestrians. Pedestrians are one of the main players in traffic systems, and they also are their most common victims. The high incident of pedestrians' accidents has led the World Health Organization (WHO) to set the reduction of traffic accidents as a priority for developing countries. Agent-based modelling and multi-agent simulation, in conjunction with systems dynamics, have open opportunities for assessing the effects of government policies, economic resources, preventive and punitive programs, educational campaigns, cultural changes, and rate of accidents, to model the individual and group behavior in pedestrian modelling. We present an analysis of the literature to identify the gaps and opportunities to develop better quantitative models of pedestrian systems, including the identification of the factors and variables that affect their functionality.

Keywords-Pedestrian Systems, agent-based Modelling, pedestrian Modelling.
\end{abstract}

Digital Object Identifier

(DOI):http://dx.doi.org/10.18687/LACCEI2016.1.1.265

ISBN: 978-0-9822896-9-3

ISSN: 2414-6390

$14^{\text {th }}$ LACCEI International Multi-Conference for Engineering, Education, and Technology: "Engineering Innovations for Global Sustainability", 20-22 July 2016, San José, Costa Rica. 


\title{
Pedestrian Systems Modelling: Opportunities and Challenges
}

\author{
Martha A. Centeno, $\mathrm{PhD}^{1}$, Lindsay Álvarez Pomar, $\mathrm{MSc}^{2}$, and German Méndez Giraldo, $\mathrm{PhD}^{1}$ \\ ${ }^{1}$ Universidad del Turabo, Puerto Rico; Universidad Distrital Francisco José de Caldas, Colombia; centenom1@ suagm.edu, \\ gmendez@udistrital.edu.co. \\ ${ }^{2}$ Universidad Distrital Francisco José de Caldas, Colombia, lalvarez@udistrital.edu.co
}

\begin{abstract}
Designing pedestrian systems have been a challenge due to the lack of effective tools and methods to model the autonomous behavior of pedestrians. Pedestrians are one of the main players in traffic systems, and they also are their most common victims. The high incident of pedestrians' accidents has led the World Health Organization (WHO) to set the reduction of traffic accidents as a priority for developing countries. Agent-based modelling and multi-agent simulation, in conjunction with systems dynamics, have open opportunities for assessing the effects of government policies, economic resources, preventive and punitive programs, educational campaigns, cultural changes, and rate of accidents, to model the individual and group behavior in pedestrian modelling. We present an analysis of the literature to identify the gaps and opportunities to develop better quantitative models of pedestrian systems, including the identification of the factors and variables that affect their functionality.
\end{abstract}

Keywords-Pedestrian Systems, agent-based Modelling, pedestrian Modelling.

\section{INTRODUCTION}

People are active participants in many systems, natural and engineered systems. Their behavior impact the performance of these systems, specially engineered systems such as service, transportation, and traffic systems. However, modelling peopleôs behavior has been a challenge, one that must be overcome if we are to meet the challenges of large cities in the $21^{\text {st }}$ century. We need tools and methods to be able to assess the movement of people, as a group and as individuals, so we can better plan, design, and redesign pedestrian systems [1].

Pedestrians are one of the main players in traffic systems, and they are also the most common victim of these systems. In fact, the impact of pedestrian accidents is so significant that the World Health Organization (WHO) set the reduction of traffic accidents as a priority for developing countries [2], which raises the urgency of developing more comprehensive methods and models for pedestrian modelling.

Pedestrian modelling emulates the movement of people, their interactions with other people, and their interaction with the environment in which they move. Pedestrian modelling plays a major role in assessing design alternatives of a variety of systems and processes, such as emergency protocols, evacuation routes, and flow of pedestrians inside buildings (airports, stadium, metro stations, walkways, etc.) [3]. Current standards for the design of pedestrian mobility schemes do not consider pedestrians as autonomous entities; rather, they limit themselves to assessing design alternatives and dimensioning issues. These standards or guidelines have ignored pedestrian behavior. In fact, most academic research has focused on studying the flow of pedestrians at specific places in a city, without regards to its relations to other elements of a mobility system.

One cause for the difficulty of properly modelling pedestrians reside on the differences between pedestrian travel and other forms of travel. Pedestrians are not restricted to one dimensional flows, like vehicular travel, nor are they restricted to a finite discrete set of decision points (nodes); hence, pedestrian models are not amenable to classical network models. Pedestrians move freely, and at a momentôs notice, generating plausibly an infinite number of routes for each pedestrian [4]. Another reason is, in part, the lack of political will. Cities underwent a drastic transformation in the $20^{\text {th }}$ century. From being places friendly to pedestrians, they became places friendly to motor vehicles, to the point that they have almost no infrastructure for pedestrian travel and mobility as reflected by the fact the of 15 cities studied by the Latin American Observatory for Urban Mobility, less than $0.5 \%$ of the road ways have some form of pedestrian priority [5].

We have reached a point in which government authorities, city planners, and civic organizations have come to the realization that pedestrians must not be an afterthought in the city planning activity. In fact, during the $51^{\text {st }}$ meeting of the Executive Council of the Pan American Health organization, the health authorities of the Americas approved an action plan regarding road safety to prevent or minimize traumas due to traffic accidents. The plan calls for countries to adapt the appropriate legislation to curve down accidents due to speed, driving under the influence, wearing of a seatbelt, wearing helmets, and using safety child seat. These plans, however, failed to explicitly call for actions to influence and modify pedestriansôbehaviors [7].

In this paper, we discuss and analyze the literature to identify the gaps and opportunities to develop better quantitative models of pedestrian systems. Our intent has been to identify a set of the most promising methods for pedestrian modelling, with special emphasis given to the behavior of pedestrian when navigating streets and at crossing points. Agent-based simulation (ABS), multi-agent simulation (MAS), and systems dynamics are two promising methods to develop more comprehensive models of pedestrian systems, including models of pedestrianôs individual and collective intelligence. There still is a need for better methods and protocols for data collection to represent the individual and interacting behavior of pedestrians.

Section II of this paper presents the generalities of research on pedestrian systems. Section III describes the methodology

Digital Object Identifier (DOI): http://dx.doi.org/10.18687/LACCEI2016.1.1.265 ISBN: 978-0-9822896-9-3 ISSN: $2414-6390$

$14^{\text {th }}$ LACCEI International Multi-Conference for Engineering, Education, and Technology: ñEngineering Innovations for Global Sustainabilityò, 20-22 July 2016, San José, Costa Rica. 
used to analyze the literature. Section IV gives the analysis and a discussion of the results. Lastly, Section V summarizes the findings and presents possible research paths based on the identified gap.

\section{Generalities of PEDESTRIAN Systems RESEARCH}

Research on pedestrian systems and modelling has been done from several perspectives that include psychology, statistics, and civil engineering. Regardless of the perspective, mobility decision making has focused primarily on the needs for vehicular movements, based on historical accidents data, failing to include the behaviour of pedestrians and their autonomous logic to navigate a pedestrian system [6]. Despite the high incidence of accidents [9], in many cities, pedestrians have become second class users of pedestrians systems, who have not only been ignored during the design of these systems, but also have been blamed when accidents occurred. Even when they are not ignored, pedestrians are assumed to be wellbehaved and identical entities. This must change if we are to affect the traffic accidents statistics. Although some developed countries began to show improvements in this regard, there is much more that needs to be done. The world cannot continue to have traffic accidents as the \#9 killer in the world [9]. It should not even be in the top 100 !

Another focus of pedestrian modelling research has been guided by the impact traffic accidents have on the victims, their families, and society at large. Traffic accidents result in higher demand of healthcare services and higher costs of treatments because they fall under the category of ñemergencyò treatments. In 2005, in Brazil, expenses related to the treatment of victims of traffic accidents reached $\$ 10$ billion dollars. To put this into perspective, $\$ 10$ billion dollars represented $1.2 \%$ of Brazilôs gross domestic product (GDP) [9]. Furthermore, not only traffic accidents incur real costs, but they also generate opportunity losses. For example, in Colombia, up to 2004, costs due to traffic accidents represented anywhere from $1.25 \%$ to $2.5 \%$ of the GDP, and the productivity losses amounted to approximately 201,000 person-years [10].

To address this issue, regional and worldwide organizations have sponsored research and disseminated studies to highlight the importance of designing streets and traffic systems that 1) are friendly to pedestrians, and 2) encourage the habit of pedestrian travel (walking) [9].

Most research addresses this issue based on historic information, and some research efforts are conducted in isolation, to address a specific, narrow issue, and not necessarily to build upon previous advances in pedestrian systems ([4], [11], [12]). There is a need to frame pedestrian modelling research as an integrated effort in which we include a reactive perspective as well as a preventive perspective. To adequately integrate the former, we need to include historic records, whereas for the latter, we need to understand the psychology of pedestrian behaviour, and develop means to integrate it in quantitative planning models.

Pedestrian traffic has been mostly studied from three perspectives:

1) Intelligent transportation systems (ITS).
2) Evacuation protocols.

3) Street flow and movement.

ITS seeks to create artifacts, algorithms and infrastructure to affect pedestrian systems. For example, research has focused on pedestrian facial identification, increase pedestrian security, and reduce the magnitude of traffic accidents.

Evacuation protocols seek to develop design methods and strategies to minimize the time needed to evacuate areas of high pedestrian flux.

Street flow and movement research falls into one of two categories: analysis of flow and walking patterns. For the former, the research is based on hydrodynamic, and for the latter, the research is divided into space-related and behaviour related. For space related efforts, research is based on physics analysis and cellular automata cells, whereas for behaviour, they have used statistical analysis, statistical modelling and multi-agent modelling.

Fig. 1 show the hierarchy and focus of the research on pedestrian systems.

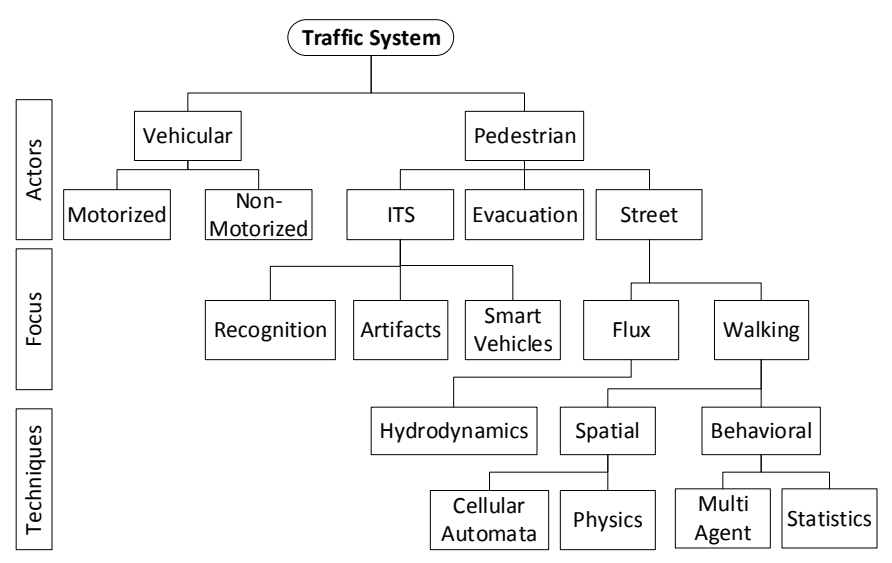

Fig. 1 Hierarchy and focus of research.

\section{METHODOLOGY}

Making a contribution to the state-of-the-art in pedestrian modelling requires a clear identification of the gaps left behind by previous research, and then it is necessary to identify the reasons for the existence of such gaps. The latter analysis leads to proposing potential strategies to reduce or eliminate the gaps.

The available literature on pedestrian-related studies is very extensive. Thus, we decided to conduct our effort in two phases. In this sense, we use a methodology similar to that presented in [8], which resembles stratified experimental sampling. Phase 1 is limited to searches on the Proquest and Science Direct databases. These two databases were chosen as they are heavily oriented towards engineering publications. Phase 2 of our effort is being guided by the findings of Phase 1 to include other databases, and to expand the time window; however, this phase is still underway, and we expect to publish comprehensive findings in a journal article.

Phase 1 consisted of 3 tasks as proposed in [8]: exhaustive searches, review of abstracts, review of full article. Exhaustive searches where conducted using as keywords ñpedestrianò, ñpedestrian modellingò, and ñpedestrian-simulationò. The

$14^{\text {th }}$ LACCEI International Multi-Conference for Engineering, Education, and Technology: ñEngineering Innovations for Global Sustainabilityò, 20-22 July 2016, San José, Costa Rica. 
logical expression was set for these keywords to appear in [<title> OR <abstract> OR <keywords>].

We developed a table of seven fields, which we implemented in Excel for no particular reason, and it is shown in Table I. The domain for PM and SM is a set of six members: qualitative, mathematical Modelling, statistical analysis, statistical Modelling, simulation, and other. For the cases where ñsimulationò was either PM or SM, we further identified it according to: multi-agent, automata, continuous, discrete, Newton, and floor field model.

TABLE I

SCHEMA FOR ARTICLE RECORDS

\begin{tabular}{|l|l|}
\hline Fields & Description \\
\hline Year & Year of publication \\
\hline Author & List of authors \\
\hline Title & Article title \\
\hline Keyword & Set of keywords \\
\hline PM & Primary method used \\
\hline SM & Secondary method used \\
\hline Focus & Simulation perspective and focus used in the study \\
\hline DataType & Binary; $1=$ uses real world data; $0=$ otherwise \\
\hline
\end{tabular}

\section{ANALYSIS AND DISCUSSION OF RESULTS}

According to the methodology, we conducted searches on two scientific databases: ProQuest and Science Direct. Then performed a two-step review to classify and screen the articles. The review of the abstracts enabled us to identify that indeed the articles were significant to pedestrian modelling. Once the article was flagged as significant, the full text was reviewed to identify its focus, methods used, and the type of data used in the study. The latter helped us determine if there were any trends to solve specific problems rather than developing a body of knowledge for pedestrian modelling.

From step 1 of Phase I (the searches for each keyword), we obtained the results shown in Table II. As you can see, for these keywords, approximately $91 \%$ of the articles found in science direct are refereed journal articles, on average; whereas about $55 \%$ fall in this category, on average, for ProQuest.

For step 2, we reviewed the titles and abstracts of the first 300 articles in order of importance, giving special attention to those that had ñstreetò related contents. The reduction on sample size was guided by various facts. First, although evacuations many a times make use of streets, they take place under conditions of duress and do not represent a ñormalò pedestrian behavior. These articles described efforts pertaining to close spaces and seek to minimize the evacuation time; hence, we did not look at articles pertaining to evacuations any further. Second, anything that was mostly focused on ITS was removed from further consideration because the amount of pedestrian behavior in those efforts was either non-existent or minimal. Most efforts in ITS have been focused on the development of artifacts that seek to preserve the life of those on board of a vehicle. Third, articles that based their analyses on qualitative methods were also removed from further consideration because although they include important behavioral information, they do not address the issue of explicit representation of such behaviors. Only a handful of these were retained because they show meaningful results.
For step 3, we reviewed the full text of the remaining 117 articles to extract the information of Table I. We found that $86.3 \%$ of the reviewed articles were published after 2005, with $61 \%$ of them published after 2010 . In regards to the primary methods used, $32 \%$ used simulation, $26 \%$ used mathematical modeling, and $25 \%$ use qualitative methods. On the other hand, qualitative methods are the most used as a secondary method (27\%) as shown in Table III. These results are graphically reflected in Fig. 2. It is important to point out that some of the articles used more than two of these methods. Of those articles that used simulation, $28 \%$ used multi-agents, and 23\% used cellular automata. $38 \%$ of the simulation articles used real world data as shown in Table IV. 55\% of those efforts that used simulation as PM, used mathematical modeling as the SM, and $32 \%$ use a qualitative method as SM as shown in Table V. It is evident that planners and researchers have realized that pedestrians must be an integral part of designing traffic systems.

TABLE II

RESULTS OF SEARCHES

\begin{tabular}{|l|c|c|c|c|}
\hline \multirow{2}{*}{} & \multicolumn{2}{|c|}{$\begin{array}{c}\text { ProQuest } \\
\text { (totals) }\end{array}$} & \multicolumn{2}{c|}{$\begin{array}{c}\text { Science Direct } \\
\text { (totals) }\end{array}$} \\
\hline Keyword & Search & Journal & Search & Journal \\
\hline Pedestrians & 8173 & 3617 & 30460 & 27176 \\
\hline Pedestrians modeling & 429 & 251 & 10268 & 9497 \\
\hline Pedestrians simulation & 306 & 204 & 7804 & 7235 \\
\hline
\end{tabular}

TABLE III

PRIMARY AND SECONDARY METHODS

\begin{tabular}{|l|c|c|c|c|}
\hline \multirow{2}{*}{\multicolumn{1}{|c|}{ Method }} & \multicolumn{2}{c|}{ PM } & \multicolumn{2}{c|}{ SM } \\
\cline { 2 - 5 } & Total & $\%$ & Total & $\%$ \\
\hline Simulation & 38 & $32 \%$ & 19 & $16 \%$ \\
\hline Math Modelling & 30 & $26 \%$ & 24 & $21 \%$ \\
\hline Qualitative & 29 & $25 \%$ & 32 & $27 \%$ \\
\hline Statistical Analysis & 16 & $14 \%$ & 28 & $24 \%$ \\
\hline Statistical Modelling & 4 & $3 \%$ & 3 & $3 \%$ \\
\hline Other & 0 & $0 \%$ & 3 & $3 \%$ \\
\hline none & & & 8 & $7 \%$ \\
\hline Grand Total & 117 & $100 \%$ & 117 & $100 \%$ \\
\hline
\end{tabular}

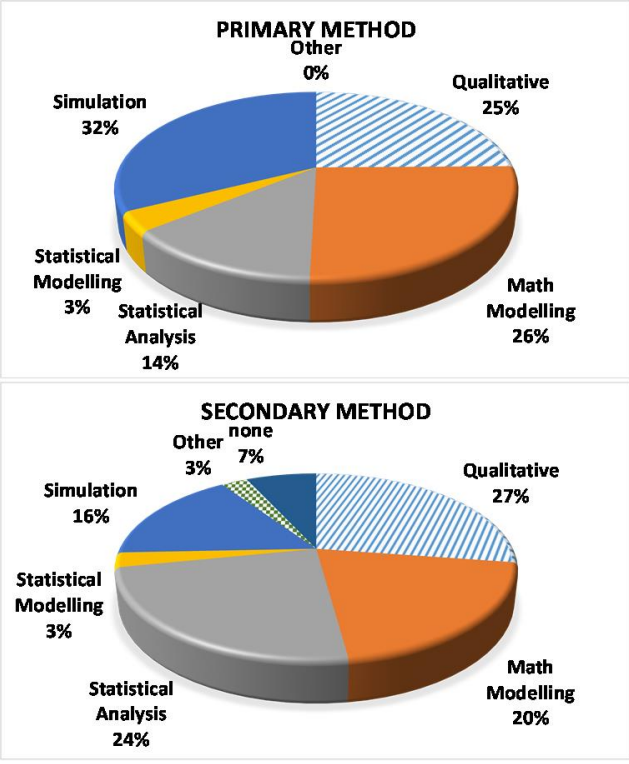

Fig. 2 Comparison of method usage.

14 ${ }^{\text {th }}$ LACCEI International Multi-Conference for Engineering, Education, and Technology: ñEngineering Innovations for Global Sustainabilityò, 20-22 July 2016, San José, Costa Rica. 
TABLE IV

SiMULATION APPROACHES

\begin{tabular}{|c|c|c|c|c|}
\hline $\begin{array}{c}\text { Multi- } \\
\text { agent }\end{array}$ & Automata & Continuous & Discrete & $\begin{array}{c}\text { Newton / } \\
\text { floor field } \\
\text { model }\end{array}$ \\
\hline 13 & 11 & 7 & 7 & 9 \\
\hline $28 \%$ & $23 \%$ & $15 \%$ & $15 \%$ & $19 \%$ \\
\hline
\end{tabular}

TABLE V

SECONDARY TECHNIQUE FOR SIMULATION APPROACHES

\begin{tabular}{|c|c|c|c|c|}
\hline Qualitative & $\begin{array}{c}\text { Mathematical } \\
\text { Modeling }\end{array}$ & $\begin{array}{c}\text { Statistical } \\
\text { Analysis }\end{array}$ & $\begin{array}{c}\text { Statistical } \\
\text { Modeling }\end{array}$ & Other \\
\hline 12 & 21 & 3 & 1 & 1 \\
\hline $32 \%$ & $55 \%$ & $8 \%$ & $3 \%$ & $3 \%$ \\
\hline
\end{tabular}

Qualitative methods are being combined with simulation and mathematical model more and more. Efforts that have used qualitative methods are beginning to yield comprehensive information in regards to how pedestrians navigate pedestrian systems. Among the most successful strategies are surveys, such as the one in [13], field studies, such as the one in [14], [15], [16], and [17]. In addition, some of these efforts have given us methodologies to develop surveys and field studies protocols, such as [18] and [19].

Significant progress has been made to represent objects that can act individually as well as part of a class. Particularly, in the case of simulation, the advent of agent-based simulation (ABS) has given way to multi-agent simulation (MAS), in which agents possess some form of individual and community intelligence. In this sense, MAS is an extension of ABS. The focus of efforts that have used MAS have been on analyzing the dimensioning of infrastructure, or positioning artifacts, that are friendlier to pedestrians. Nevertheless, these efforts have managed to model behavioral information such as the formation of groups ([20], [21]), pedestrian strength ([22], [23]), environmental factors [24], and individual characteristics such as gender, vision range, knowledge of the neighborhood, and the urgency of movement on the part of the pedestrian ([25], [26], [27], [28])

The mathematical modeling of pedestrians has allowed the analysis and sizing of facilities and other infrastructures, as well as global behaviors. These efforts have, for the most part analyzed pedestrian fluxes (flows), with no considerations to socio-cultural factors ([29], [30], [31], [32]). Some of these efforts have integrated simulation models, such as those presented in [33], [34], and [35]. Only one of these efforts included the use real world data [15].

From the literature, we can say that significant first steps have been taken to design traffic systems that treat the pedestrian component as equal to the vehicular component, but there remain significant challenges ahead. Among these challenges are:

- Lack of data that captures the movement, the behavior, and the decision making of pedestrians as they sojourn a traffic system.

- Lack of effective methods and means to measure pedestrianôs movement flows and pedestrian safety. In other words, we need new means to data needed.
- Little or no government units that are focused on studying and understanding the role of pedestrians, their behavior, and collective intelligence in designing better traffic systems.

- Lack of comprehensive programs to train pedestrians to be responsible agents in a traffic system.

- Lack of legislative and personnel infrastructures to enforce traffic laws that apply to vehicles as well as to pedestrians.

These challenges give rise to a series of research activities and opportunities. For example, to build upon the efforts that have used qualitative methods, it is now necessary to continue some of these studies or start new one so that we can develop generalizations of the factors that influence pedestrian behavior. Another thing that still needs to happen is the conversion of information gathered, and future generalizations, into some form of data-based or probabilistic models, so that these models can be part of simulation and mathematical models.

Efforts that have used mathematical model may need to be combined with either some qualitative method or embed a simulation model, or vice versa, to model social intervention scenarios.

Efforts that have used MAS now need to be extended so that new models can be built to evaluate pedestrian intervention strategies and individual and collective pedestrian behavior modification strategies, so that city planners and other decision and policy makers can develop plan and approve projects that lead to a reduction in traffic accidents. In addition, some of the efforts have no given evidence that the representation used for some characteristics is based on some form of statistical analysis. We need to develop and conduct more field studies so that we can unequivocally assess the importance of salient variables in pedestrian behavior, as individuals and as part of specific social groups. This could lead to explicitly identify socio-cultural differences that affect pedestrian behavior.

For both simulation and mathematical modeling, we need to develop data collection mechanism that would enable the building of a database of raw data that could lead to enriching models with more details in regards to pedestrian behavior.

There are also opportunities in the legislative front. If pedestrians are to be treated as components of a traffic system with equal weight to vehicles, then pedestrians need to be ñtrainedòin an equivalent fashion as to a driver. In other words, all citizens need to be taught the rules and regulations of being a pedestrian that interacts with vehicles. Order reduces the number of accidents involving pedestrians, but human beings tend to be opportunistic entities, a behavior that tends to lead to disruptions in the whole system. Educational programs of these type need to be paired with a set of laws that when broken penalized pedestrians. An example of these laws is a law that penalizes jaywalking.

Another legislative opportunity is ensuring pedestriansô ways are safe from both the perspective of vehicles (clear signs, laws. Driversô training, protected ways, etc.) and from the perspective of other pedestrians (well lighted walkways and

$14^{\text {th }}$ LACCEI International Multi-Conference for Engineering, Education, and Technology: ñEngineering Innovations for Global Sustainabilityò, 20-22 July 2016, San José, Costa Rica. 
adequate policing to minimize the likelihood of thefts and assaults).

\section{SUMMARY}

We have described the analysis of the literature on pedestrian modelling for phase 1 . We conducted searches on scientific databases, and performed a two-step review to classify and screen the articles. Results indicate that the method most commonly used to study pedestrian systems has been simulation. Within this, the most common approach has been multi-agent simulation.

The review has revealed that to continue advancing the body of knowledge in this area, we need to design and conduct more field studies so that we can unequivocally assess the importance of salient variables in pedestrian behavior, as individuals and as part of specific social groups.

In summary, future research may focus on:

1) Conducting research to so that we can develop generalizations of the factors that influence pedestrian behavior. We need to find a way to correlate the results of the various efforts in which qualitative methods are used so that the behavioral information they elicit is properly kept and made accessible.

2) Collecting and disseminating qualitative data so that appropriate conversions of the information gathered, is done, seeking generalizations that can be part of simulation and mathematical models.

3) Framing pedestrian modelling research as an integrated effort in which we include a reactive perspective as well as a preventive perspective, i.e. evaluate intervention programs to modify pedestrian behavior.

4) Understanding the psychology of pedestrian behavior, and to develop means to integrate it in planning models.

5) Integrating a pedestrian modeling module in planning models to allow for the generation of pedestrianfriendly and flexible Pedestrian systems.

6) Developing new social programs via legislation.

Mathematical model may need to be combined with either some qualitative method or embed a simulation model, or vice versa, to model social intervention scenarios. New MAS models have to be built to enable evaluation pedestrian intervention strategies and individual and collective pedestrian behavior modification strategies.

The review and analysis presented here, and the on-going phase, has served as the basis to another effort by [36], who has recently completed her doctoral dissertation work by developing a model of pedestrian collective intelligence. The model enables the measurement of the global influence of decisions made at different levels. This model is a first step towards quantitative modelling of open social systems. Future articles will discuss the model and other results.

Digital Object Identifier: (to be inserted by LACCEI).

\section{REFERENCES}

[1] L. Alvarez-Pomar, G. Méndez-Giraldo, and N. Martins-Goncalves, ñPedestrian system design,ò Revista Tecnura, vol. 18 (Edición especial doctorado), 124ï 135. doi: 10.14483/udistrital.jour.tecnura. 2014. DSE1. a11, pp. 124-135, December 2014.

[2] WHO, World Report on Road Traffic Injury Prevention, Chapter 4

[3] S. P. Hoogendoorn, ñPedestrian travel behavior modelling,ò In Proceedings of the $10^{\text {th }}$ International Conference on Travel Behavior Research, Session XXX, August 2003.

[4] S. P. Hoogendoorn, and P. H. L. Bovy, ñPedestrian travel behavior modelling,ò Networks and Spatial Economics, vol. 5, no. 2, pp. 193 ï 216. doi:10.1007/s11067-005-2629-y, 2005.

[5] J. Kogan, S. Azán, M. E. Miquilena, and E. Vasconcellos, Observatorio de Movilidad Urbana para América Latina, Andean Foment Corporation, 2010.

[6] G. Ulloa, ñEl tránsito vehículo-peatonal desde un punto de vista psicocultural,ò Revista Costarricesnse de Psicología, vol. 30, nos. 45-46, $\mathrm{pp} /$ 95-112, 2011.

[7] OPS-OMS Plan De Acción De Seguridad Vial (Vol. 16),2011.

[8] S. C. Brailsford, P. R. Harper, B. Patel, and M. Pitt, ñAn analysis of the academic literature on simulation and modelling in healthcare,òJournal of Simulation, no. 3, pp. 130ї 140, 2009.

[9] OCDE, Peatones: seguridad vial, espacio urbano y salud. Retrieved from http://www.internationaltransportforum.org/ pub/ pdf/ 11Pedestrian SumES. Pdf, Research report, OECD, 2011.

[10] Ministerio de transporte de Colombia, ñHacia una Nueva Cultura de Seguridad Vial,ò Plan Nacional de Seguridad Vial 2004-2006 Bogotá: Ministerio del Transporte.

[11]A. Galderisi, and A. Ceudech, ñSoft mobility and pedestrian networks in urban areas,ò TeMaLab Journal of Mobility, Land Use and Environment, vol. 3, SP March 2010, pp. 21-28, 2010.

[12] I. N. Franco, ñSegundo Estudio de Comportamiento y Seguridad Vial,ò Bogotá: Fondo de Prevención Vial, Retrieved on June $20^{\text {th }}$, 2015, from http://fpv.org.co/images/repositorioftp/fpv-ii estudio vial.pdf, 2012.

[13] A. Dommes, M. A. Granié, M. S. Cloutier, C. Coquelet, F. HugueninRichard, F. ñRed light violations by adult pedestrians and other safetyrelated behaviour s at signalized crosswalks.òJournal of Accident Analysis \& Prevention, vol. 80, pp. 67ï 75. doi:10.1016/j.aap.2015.04.002, 2015.

[14] A. Tom, and M. A. Granié, M.-A. (2011). Gender differences in pedestrian rule compliance and visual search at signalized and un-signalized crossroads. Journal of Accident Analysis and Prevention, 43(5), 1794ї 1801. doi:10.1016/j.aap.2011.04.012

[15] M. M. Hamed, ñAnalysis of pedestriansôbehavior at pedestrian crossings,ò Safety Science, vol. 38, no. 1, pp. 63ї 82, doi:10.1016/ S0925-7535 (00)00058-8, 2001.

[16] M. J. M. Sullman, M. E. Gras, S. Font-Mayolas, L. Masferrer, M. Cunill, and M. Planes, ñThe pedestrian behaviour of Spanish adolescents, òJournal of Adolescence, vol. 34, no. 3, pp. 531ї 9. doi:10.1016/ j.adolescence. 2010.05.011, 2011.

[17]C. Milligan, R. Poapst, and J. Montufar, ñPerformance measures and input uncertainty for pedestrian crossing exposure estimates, ò Accident Analysis and Prevention, vol. 50, pp. 490ï 498. doi:10.1016/j.aap.2012.05.024.

[18]E. Papadimitriou, ñTheory and models of pedestrian crossing behaviour along urban trips,ò Transportation Research Part F: Traffic Psychology and Behavior, vol. 15, no. 1, pp. 75ï 94. doi:10.1016/j.trf.2011.11.007, 2012.

[19]T. Robin, G. Antonini, M. Bierlaire, and J. Cruz, J. ñSpecification, estimation and validation of a pedestrian walking behavior model,ò Transportation Research Part B: Methodological, vol. 43, no. 1, pp. 36ї 56. doi:10.1016/j.trb.2008.06.010, 2009.

[20]F. Qiu, and X. Hu, ñModelling dynamic groups for agent-based pedestrian crowd simulations,ò In Proceedings of the 2010 IEEE/WIC/ACM International Conference on Web Intelligence and Intelligent Agent Technology, pp. 461ï 464, IEEE, doi:10.1109/WI-IAT.2010.9, 2010a.

[21]F. Qiu, and X. Hu, ñModelling group structures in pedestrian crowd simulation,òSimulation Modelling Practice and Theory, vol. 18, no. 2, pp. 190ї 205, doi:10.1016/j.simpat.2009.10.005, 2010b.

[22] H. Gotoh, E. Harada, and E. Andoh, ñSimulation of pedestrian contra-flow by multi-agent DEM model with self-evasive action model,ò Safety Science, vol. 50, no. 2, pp. 326ï 332. doi:10.1016/j.ssci.2011.09.009, 2012.

$14^{\text {th }}$ LACCEI International Multi-Conference for Engineering, Education, and Technology: ñEngineering Innovations for Global Sustainabilityò, 20-22 July 2016, San José, Costa Rica. 
[23] N. Gaud, S. Galland, F. Gechter, V. Hilaire, and A. Koukam, ñHolonic multilevel simulation of complex systems: Application to real-time pedestrians simulation in virtual urban environment,ò Simulation Modelling Practice and Theory, vol. 16, no. 10, pp. 1659ї 1676, doi:10.1016/ j.simpat.2008.08.015, 2008.

[24] G. D. P. Aschwanden, T. Wullschleger, H. Müller, and G. Schmitt, ñAgent based evaluation of dynamic city models, Ò Automation in Construction, vol. 22, pp. 81ї 89, 2012.

[25] W. L. Wang, S. M. Lo, S. B. Liu, and J. Ma, ñOn the use of a pedestrian simulation model with natural behavior representation in metro stations. Procedia Computer Science, vol. 52, pp. 137ï 144. doi:10.1016/ j.procs.2015.05.048, 2015.

[26] Toyama, M. C., Bazzan, A. L. C., \& Silva, R. (2006). ñAn agent-based simulation of pedestrian dynamics $\mathbb{E}$ from lane formation to auditorium evacuation,ò In Proceedings of the fifth international joint conference on AAMAS- ACM, 108ї 110, doi: 10.1145/1160633.1160647, 2006.

[27]C. Hollmann, P. J. Lawrence, and E. R. Galea, ñIntroducing emotion modelling to agent-based pedestrian circulation simulation,ò Pedestrian and Evacuation Dynamics, R. D. Peacock et al (eds.), pp. 799-803, DOI 10.1007/978-1-4419-9725-8-77. 2011.

[28] A. Banos, and A. Charpentier, ñSimulating pedestrian behavior in subway stations with agents.ò In Proceedings of the 4th European Social Simulation Association, 2007, pp. 611ï 621, Toulouse, France.

[29] R. Galiza, and L. Ferreira, ñA methodology for determining equivalent factors in heterogeneous pedestrian flows,ò Computers, Environment and Urban Systems, vol. 39, May, pp. 162ï 171. doi:10.1016/ j.compenvurbsys.2012.08.00, 2013.
[30] A. Seyfried, B. Steffen, and T. Lippert, ñBasics of modelling the pedestrian flow,ò Physica A: Statistical Mechanics and Its Applications, vol. 368, no. 1, pp. 232ï 238. doi:10.1016/j.physa.2005.11.052, 2005.

[31] M. Muramatsu and T. Nagatani, ñJamming transition in two-dimensional pedestrian traffic,ò Physica A: Statistical Mechanics and Its Applications, vol. 275, $281 і ̈$ 291, 2000.

[32] J. Liang, Y. Zhang, and J. Yang, ñAn extended small-grid lattice gas model for pedestrian counter flow,ò Procedia Engineering, vol. 62, 2013, pp. 501ї 508. doi:10.1016/j.proeng.2013.08.093, 2013.

[33] A. Kneidl, D. Hartmann, and A. Bormann, ñA hybrid multi-scale approach for simulation of pedestrian dynamics, ò Transportation Research Part C: Emerging Technologies, vol. 37, December, pp. 223ї 237. doi:10. 1016/j.trc.2013.03.005, 2013.

[34] J. Buisson, S. Galland, N. Gaud, M. Gonçalves, and A. Koukam, ñRealtime collision avoidance for pedestrian and bicyclist simulation: A smooth and predictive approach.ò In The 2nd International Workshop on Agentbased Mobility, Traffic and Transportation Models, Methodologies and Applications (ABMTRANS), Procedia Computer Science, vol. 19, pp. 815ï 820, 2013.

[35] M. Nasir, C. P. Lim, S. Nahavandi, and D. Creighton, ñA genetic fuzzy system to model pedestrian walking path in a built environment,ò Simulation Modelling Practice and Theory, vol. 45, June, pp. 18-34, 2014.

[36]L. Alvarez-Pomar, Modelo de Inteligencia Colectiva de los Sistemas Peatonales, Doctoral Dissertation, Universidad Distrital, Bogota, Colombia, 2016.

14 ${ }^{\text {th }}$ LACCEI International Multi-Conference for Engineering, Education, and Technology: ñEngineering Innovations for Global Sustainabilityò, 20-22 July 2016, San José, Costa Rica. 